\title{
Prática de cultivo e uso de plantas domésticas em diferentes cidades brasileiras
}

\author{
Cultivation and use of domestic plants in different Brazilian cities
}

\author{
Juliana de Mello Botelho ${ }^{\mathrm{I}}$ Ana Paula do Nascimento Lamano-Ferreira ${ }^{\text {II }}$ \\ Mauricio Lamano Ferreira ${ }^{\mathrm{F}^{*}}$
}

\section{RESUMO}

\begin{abstract}
O cultivo de plantas tem uma relação com o homem desde as primeiras organizações humanas. $O$ uso de plantas em quintais é uma tradição que tem passado de geração a geração em determinadas localidades. $O$ objetivo deste trabalho foi levantar a ocorrência de quintais domésticos em diferentes cidades de três estados brasileiros, além de conhecer uso das plantas cultivadas para fins alimentícios, medicinais e ornamentais. Este estudo foi realizado nas cidades de três estados brasileiros: Mato Grosso (Cuiabá e Cáceres); Sergipe (Aracajú e Lagarto) e Paraná (Curitiba e Campo Mourão), localizados em diferentes regiões do país (Centro Oeste, Nordeste e Sul; respectivamente). Foram realizadas entrevistas semi-estruturadas com moradores das capitais e cidades interioranas. Os dados foram avaliados quanto ao conhecimento popular em relação ao uso das plantas pelas populações, bem como as estruturas vegetais utilizadas. A maior parte dos entrevistados possuía quintais com plantas cultivadas. Foi observado um maior cultivo e uso de plantas alimentícias pelos entrevistados. As pessoas das cidades interioranas apresentaram maior hábito de cultivar plantas quando comparadas às pessoas residentes nas capitais dos estados. Dentre os órgãos da planta mais citados, a folha se destacou na maioria dos entrevistados. Assim, conclui-se que há prática de cultivo de plantas nos municípios estudados, entretanto, o uso varia de uma região para outra.
\end{abstract}

Palavras-chave: quintais, plantas ornamentais, plantas medicinais, plantas alimentícias, sustentabilidade.

\section{ABSTRACT}

There is a relationship between cultivation of plants and mankind since the earliest human organizations. The use of plants in home gardens is a tradition that has been passed down from generation to generation in certain localities. The aim of this research was to verify the occurrence of home gardens in different cities of three Brazilian states, besides knowing the use of cultivated plants for feed, medicinal and ornamental purposes. This study was carried out in cities of three Brazilian states: Mato Grosso (Cuiabá and Cáceres), Sergipe (Aracaju and Lagarto) and Paraná (Curitiba and Campo Mourão) located in different regions of the country (Midwestern, Northeastern and Southern). Semi-structured interview were performed with residents of the state capital and countryside. Data were evaluated for popular knowledge regarding the use of plants and plant structure. Most interviewers have gardens with plants. Greater cultivation and use of food plants was observed by the interviewees, with the exception of Aracaju city. Residents in countryside had higher habit to cultivate plants when compared to people residing in the state capitals. Among the plant organs most cited the leaf stood out. Thus, it is concluded that there is a great practice of cultivating plants in the studied cities, however the use of plants varies from one region to another.

Key words: homegardens, ornamental plants, medicinal plants, food plants, sustainability.

\section{INTRODUÇÃO}

Desde os primeiros momentos da organização humana até o presente, o cultivo de plantas tem uma estreita relação com a população. Os recursos vegetais podem servir como fonte de alimento (SIVIERO et al., 2011), remédios (PAULA \& CRUZ-SILVA, 2010), ornamentação (QUEIROZ \& LAMANO-FERREIRA, NO PRELO), religião (FRANCO et al., 2011), dentre outras finalidades (ALMADA, 2010). A relação da população humana com as plantas em quintais é uma tradição que tem passado de geração a geração em determinadas

I Departamento de Saúde, Universidade Nove de Julho (UNINOVE), Campus Memorial, Av. Adolfo Pinto, 109, Água Branca, 05001-100,

São Paulo, SP, Brasil. E-mail: mauecologia@yahoo.com.br.*Autor para correspondência.

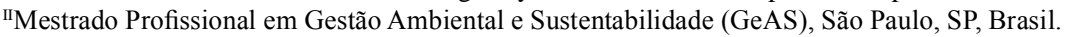


localidades, sendo denominada de etnobotânica (OLIVEIRA et al., 2009). O uso popular de plantas para qualquer finalidade é uma arte fundamentada no acúmulo de informações repassadas oralmente de pais para filhos (VIEIRA et al., 2011). Nas áreas urbanas, o hábito de cultivo de plantas em quintais está deixando de ser prioridade para as famílias, porque os quintais com espaço de terra em torno das residências estão sendo substituídos por espaços cimentados (NASCIMENTO et al., 2005). Dessa forma, as áreas urbanas das grandes cidades podem estar mais vulneráveis a perderem os quintais como espaço de plantio do que os pequenos aglomerados, devido à intensa urbanização.

De acordo com PERNA \& LAMANOFERREIRA(2014), as pesquisas realizadas sobre cultivo de plantas medicinais em cidades interioranas superam as realizadas em capitais. Nesse sentido, torna-se relevante estudar municípios urbanos, capitais e cidades interioranas, onde se espera que as famílias de cidades interioranas ainda cultivem plantas para as diversas finalidades (alimentos, remédio, ornamentação), mantendo e transmitindo informações sobre a terra, o plantio e a conservação de espécies através das gerações. Assim, levantaram-se com este trabalho as seguintes perguntas: i) As pessoas residentes nas capitais de estado apresentam um mesmo hábito em relação ao uso e cultivo de plantas em quintais que pessoas residentes em cidades interioranas? ii) Essa tendência segue um mesmo padrão em diferentes regiões brasileiras? iii) $\mathrm{O}$ uso das estruturas morfológicas das plantas se repete ao longo das diferentes cidades brasileiras? Assim, este trabalho teve como objetivos avaliar o uso de plantas em quintais domésticos em diferentes cidades de três regiões brasileiras, além de conhecer o atual quadro de conservação do germoplasma de algumas espécies de plantas alimentícias, medicinais e ornamentais cultivadas em espaços residenciais.

\section{MATERIAL E MÉTODOS}

Área de estudo

O trabalho foi realizado em três regiões brasileiras, sendo elas a região Sul, Nordeste e Centro Oeste. Em cada região, optou-se por realizar entrevistas com moradores de uma cidade interiorana e de uma capital de estado. Aescolha das capitais seguiu o princípio da aleatoriedade e a escolha das cidades interioranas seguiu um mesmo critério, sendo pré requisito ter uma população entre 50 e 100 mil habitantes.

Na região Sul, optou-se pelas cidades Campo Mourão e Curitiba. Campo Mourão é um município brasileiro localizado no interior do Estado do Paraná e possui 86.550 mil habitantes (IBGE, 2010). Curitiba é a capital do Estado, um dos três estados que compõem a região Sul do Brasil. A cidade tem uma população de 1.678.965 habitantes. Na região Nordeste, escolheu-se a cidade de Lagarto, no interior do Estado de Sergipe, que tem uma população de 94.071 mil habitantes. A capital Aracaju se localiza na região litorânea e conta com 552.365 habitantes. Na região Centro Oeste, Cáceres foi a cidade interiorana escolhida neste estudo. A cidade tem uma população de 87.942 habitantes. A capital do Estado do Mato Grosso, Cuiabá, segundo a estimativa realizada pelo IBGE (2010), a população conta com 551.098 habitantes.

População-alvo e coleta de dados

A coleta de dados ocorreu por meio de entrevistas semi-estruturadas, ou seja, uma entrevista com roteiro contendo perguntas abertas e fechadas (MARCONI \& LAKATOS, 2002). As entrevistas foram realizadas com moradores, preferencialmente mulheres, que concordaram em participar desta pesquisa, tanto nas capitais quanto nas cidades interioranas. De acordo com a literatura (SHIVA \& DALKEMAN, 1994; NASCIMENTO et al., 2006), a manutenção dos quintais e os vegetais neles cultivados, ou em qualquer outra área da residência, são de responsabilidade das mulheres. Elas decidem o que, como, onde e quando serão plantados.

A determinação dos bairros e ruas ocorreu de forma aleatória. Em cada capital, foram realizadas 200 entrevistas e, nas cidades interioranas, 50. A coleta de dados foi realizada entre os meses de agosto de 2010 e janeiro de 2012. Plantas que eventualmente não se encontraram nos quintais, mas em vasos, em algumas partes da casa, também foram consideradas.

\section{RESULTADOS E DISCUSSÃO}

Nas cidades pesquisadas, a maioria dos entrevistados apresentou mais de 61 anos de idade, com exceção a cidade de Lagarto, onde a maioria dos informantes apresentou idades entre 31 a 40 anos. Um dos fatores que pode explicar essa faixa etária encontrada é que a maioria dos entrevistados é aposentada e dedicase a maior parte do tempo aos cuidados da casa, enquanto outras faixas etárias encontram-se em outras atividades fora da residência. Outros autores encontraram maior disponibilidade e (ou) interesse de moradores acima de 40 anos de idade em participar de entrevistas, como é o caso dos trabalhos de PASA et al. (2005), FUCK et al. (2005) e CARNIELLO et al. (2010). Outra hipótese pode ser o fato de essa faixa etária (acima de 40 anos) ter uma relação mais estreita com o cultivo de plantas do que as gerações seguintes. 
Neste trabalho, mais de $68 \%$ das pessoas entrevistadas citaram que a maior fonte de obtenção de conhecimento é através dos familiares, sendo um conhecimento hereditário, que vai passando através de pais e avós para filhos. SOUZA et al. (2010), em um estudo com plantas medicinais no município de Cuiabá, MT, mencionaram que aproximadamente $90 \%$ dos entrevistados obtiveram os ensinamentos sobre o uso de plantas a partir da transmissão cultural vertical (pais para filhos), como discutido na revisão realizada por BEGOSSI (1993), fato que fortalece a hipótese de que a manutenção destas informações tem sido passada de geração a geração (VIEIRA et al., 2011) por meio de conversas familiares informais.

Nos municípios localizados nas regiões Centro Oeste e Nordeste, tanto nas capitais quanto nas cidades interioranas, a primeira palavra que mais remeteu as pessoas à lembrança de quintal foi limpeza. O que pode explicar esse fato é que nestas regiões os quintais eram predominantemente de terra batida, o que acaba sujando mais as partes internas das residências. Nos municípios da região Sul estudados, houve variação entre as respostas dos moradores da capital e do interior, sendo que em Curitiba os informantes citaram primeiramente palavras sobre ser vivo e em Campo Mourão se repetiu a tendência sobre limpeza. A referência de quintal pode variar de pessoa para pessoa, de acordo com a sua experiência de vida. Segundo SIVIERO et al. (2011), a manutenção do quintal na residência possui valores intangíveis e difíceis de serem mensurados, como o prazer de cultivar, espaço de lazer e bem estar.

Em todas as regiões pesquisadas, a maioria das residências apresentava quintais, independentes de terem o cultivo de plantas (Figura 1A). Entre as localidades (capital e interior), todas as residências

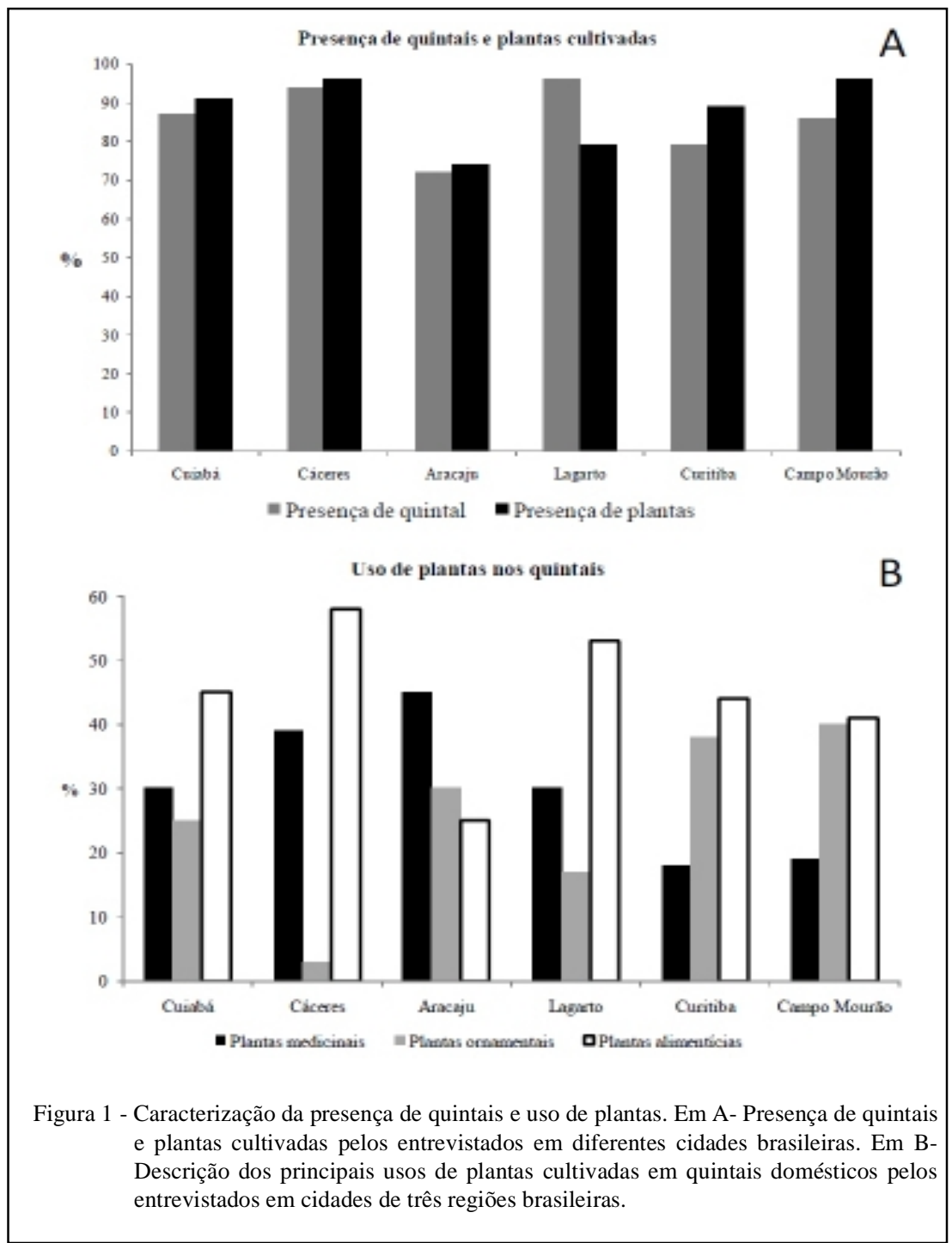

Ciência Rural, v.44, n.10, out, 2014. 
em cidades interioranas apresentaram maior quantidade de quintais. A cidade que teve a maior proporção de residências com quintais citados pelos entrevistados foi Lagarto, com 96\%, sendo Aracajú a cidade que menos apresentou residências com quintal $(72 \%)$. Em relação à região que apresentou a maior quantidade de quintais domésticos, destacase o Centro Oeste (Cuiabá: 87\%; Cáceres: 94\%). Esses espaços residenciais abrangem diversos ambientes com distintas finalidades de uso, sendo reconhecidos também como locais de conservação da biodiversidade, locais para crianças brincarem, além de ajudarem na economia familiar (BRASIL et al., 2007). Os números encontrados neste trabalho estão próximos aos da literatura, como é o caso de NASCIMENTO et al. (2005), que observaram um resultado semelhante em um trabalho realizado na cidade de Piracicaba, SP, onde $86 \%$ da população estudada afirmaram possuir quintais nas residências.

Nas cidades pesquisadas, mais de $74 \%$ dos entrevistados disseram ter plantas em seus quintais. Observou-se uma tendência a ter mais plantas cultivadas em cidades interioranas (Cáceres: 95,8\%; Lagarto: 78,5\%; Campo Mourão: 96,1\%) do que nas capitais (Cuiabá: 90,6\%; Aracaju: 73,6\%; Curitiba: 89,5\%) dos Estados estudados. Resultados similares foram encontrados na revisão bibliométrica sobre o cultivo de plantas medicinais nos últimos três anos no Brasil (PERNA \& LAMANO-FERREIRA, 2014), quando os trabalhos publicados mostram maior cultivo de plantas medicinais como também maior diversidade destas nos quintais, em cidades interioranas, quando comparadas às capitais. Os municípios de Cuiabá e Cáceres, MT, região Centro-Oeste do país, apresentaram uma maior porcentagem de cultivo, seguida por Curitiba e Campo Mourão, PR, localizadas na região Sul, e, por último, Aracaju e Lagarto, região Nordeste, cidades que apresentaram uma menor porcentagem de cultivo de plantas. Uma das dificuldades mencionadas para o cultivo de plantas pelos nordestinos, durante as entrevistas, foi a falta de água na região.

$\mathrm{O}$ uso de plantas em quintais variou dentre as cidades estudadas. Em relação às plantas medicinais, observou-se um maior uso nas cidades interioranas do que nas capitais, exceto na região nordeste do país, onde se observou o contrário (Figura 1B). As plantas ornamentais apresentaram maior uso nas capitais, com exceção da região Sul, que também contrariou a tendência de ter maior uso de plantas alimentícias nas capitais. Esses resultados vão de encontro com outras pesquisas realizadas em capitais, como em Curitiba, PR (ATHAUSOTTMANN et al., 2011) e São Paulo, SP (QUEIROZ
\& LAMANO-FERREIRA, NO PRELO) onde a maior parte das plantas cultivadas em quintais são para fins ornamentais. Já em Porto Alegre, VENDRUSCOLO \& MENTZ (2006) relatam que, mesmo sendo uma capital, a principal utilidade das plantas deste município é para fins medicinais. SIVIERO et al. (2011), em seu estudo em Rio Branco, AC, relatam a grande representação de plantas alimentícias em quintais, nesta capital. Uma possível explicação para as diferentes finalidades de plantas cultivadas em quintais pode ser por um atributo cultural, em que os tratamentos caseiros podem já não estar mais inseridos na população, sendo a medicina alopática a mais utilizada na região, além da redução nos espaços residenciais e menor distância de mercados.

A cidade de Cáceres merece destaque por ser a localidade que apresentou o maior uso de plantas para a alimentação (Figura 1B). Isso pode estar relacionado ao padrão socioeconômico das famílias. Outro fator que apoia esta hipótese é a baixa ocorrência do uso de plantas ornamentais na cidade, evidenciando a preferência dos entrevistados pelas questões alimentares. A alimentação pareceu ser o maior interesse dos entrevistados deste estudo, exceto na cidade de Aracaju, onde o maior uso foi medicinal. $\mathrm{O}$ cultivo das plantas alimentares em quintais pode ser importante na complementação da dieta alimentar de algumas populações (NACIMENTO et al., 2010; SIVIERO et al., 2011). AMARAL \& GUARIM-NETO (2008) discutem a importância do cultivo de plantas alimentares em quintais e ressaltam a importância destes vegetais na complementação nutricional, além da alternativa econômica para a suplementação de vitaminas e sais minerais essenciais para as atividades metabólicas humanas. De acordo com VIEIRA et al. (2011), as plantas medicinais surgem como um instrumento de cura para pequenas populações. ALMEIDA \& CORRÊA (2012) reportaram o uso de Pareskia aculeata para o tratamento de anemia ferropriva, câncer e osteoroporose num estudo com a população de São Gonçalo do Abaeté, MG.

Cabe enfatizar que a crescente urbanização pode gerar redução de espaços físicos residenciais ou até ausência em caso de edifícios. No entanto, nas residências visitadas nos municípios de Aracaju, Lagarto, Curitiba, Campo Mourão, Cuiabá e Cáceres, há grande ocorrência de quintais e, mesmo naquelas que não têm este espaço, há prática de cultivo de plantas em vasos.

Em todas as localidades de entrevistas, quando se tratava de planta medicinal, a parte da planta mais usada pelos entrevistados foi a folha, seguida pelo caule e raiz (Tabela 1). Estes órgãos estão constantemente expostos na maioria das 
Tabela 1 - Parte da planta medicinal utilizada para o consumo pela população entrevistada.

\begin{tabular}{|c|c|c|c|c|c|c|}
\hline & Cuiabá & Cáceres & Aracaju & Lagarto & Curitiba & Campo Mourão \\
\hline Folha & 85,2 & 85,2 & 96,6 & 96,6 & 89,6 & 94,5 \\
\hline Flor & 3,2 & $\#$ & 0,6 & 1,7 & 1,3 & $\#$ \\
\hline Broto & 1,3 & 1,7 & \# & $\#$ & $\#$ & 0,9 \\
\hline Raiz & 4,5 & 2,6 & \# & \# & 2,0 & 0,9 \\
\hline Fruto & 1,0 & 4,3 & \# & 1,7 & 1,0 & 0,9 \\
\hline Semente & 0,6 & \# & \# & \# & 1,0 & 0,9 \\
\hline Caule & 4,2 & 6,1 & 2,8 & \# & 5,1 & 1,8 \\
\hline
\end{tabular}

\# (Não citado em nenhuma entrevista).

plantas, podendo assim ser utilizados em qualquer época do ano. Outros órgãos e estruturas vegetais podem apresentar sazonalidade marcante e só estar disponível para o uso ou consumo em determinadas estações do ano, como é o caso da flor. Outros autores também identificaram as folhas como órgão de maior uso pelos entrevistados de diferentes localidades (PASA et al., 2005; SILVA et al., 2011; SOUZA et al., 2010). As folhas normalmente são utilizadas na decocção, ou seja, no preparo de bebidas medicinais, quando, após a fervura da água, se adicionam folhas a fim de extrair os princípios ativos que muitas vezes possuem metabólitos secundários e componentes voláteis que se dissolvem facilmente em água quente e apresentam efeitos benéficos a humanos (FUCK et al., 2005).

\section{CONCLUSÃO}

As cidades dos três estados brasileiros estudadas apresentaram um número expressivo de quintais domésticos com plantas cultivadas, com tendência maior nas cidades interioranas do que nas capitais. Na maioria das cidades, houve uma maior ocorrência no uso de plantas alimentícias, com exceção do município de Aracaju, onde os moradores utilizam mais plantas para fins medicinais. $\mathrm{O}$ uso das plantas alimentícias pode estar relacionado à complementação da dieta, além de ser, para algumas pessoas, uma alternativa econômica.

Com base nos dados pesquisados, sugerese que o hábito de cultivar plantas em quintais domésticos não está se perdendo devido a intensa urbanização dos últimos anos. Além disso, não foi encontrado padrão sobre as finalidades das plantas nas diferentes cidades e estados brasileiros estudados, com exceção dos municípios de Curitiba e Campo Mourão, ambos localizadas no Estado do Paraná, que seguem tendência de cultivo de plantas alimentícias, seguidas por ornamentais e medicinais. Por fim, este trabalhos identificou que a parte da planta medicinal mais utilizada em todas as cidades foi a folha, seguida por caule e raiz.

\section{REFERÊNCIAS}

ALMADA, E.D. Sociobiodiversidade Urbana: por uma etnoecologia das cidades. In: SILVA, V.A. et al. (Org.). Etnobiologia e etnoecologia: pessoas e natureza na américa latina. Recife: NUPEEA, 2010. p.39-63.

ALMEIDA, M.E.F.; CORRÊA, A.D. Utilização de cactáceas do gênero Pareskia na alimentação humana em um município de Minas Gerais. Ciência Rural, v.42, n.4, p.751-756, 2012. Disponível em: <http://www.scielo.br/pdf/cr/v42n4/a11112cr5075. pdf $>$. Acesso em: 13 jun. 2013.

ALTHAUS-OTTMANN, M.M. et al. Diversidade e uso de plantas cultivadas nos quintais do Bairro Fanny, Curitiba, Paraná, Brasil. Revista Brasileira de Biociências, v.9, n.1, p.39-49, 2011.

AMARAL, C.N.; GUARIM-NETO G. Os quintais como espaços de conservação e cultivo de alimentos: um estudo na cidade de Rosário Oeste (Mato Grosso, Brasil). Boletim do Museu Paraense Emílio Goeldi Ciências Humanas, v.3, n.3, p.329-341, 2008. Disponível em: <http://scielo.iec.pa.gov.br/pdf/bmpegch/ v3n3/v3n3a04.pdf $>$. Acesso em: 02 ago. 2012.

BEGOSSI, A. Ecologia humana: um enfoque das relações homemambiente. Revista Interciência, v.18, n.3, p.121-132, 1993. Disponível em: <http://www.interciencia.org/v18_03/art01/>. Acesso em: 10 nov. 2012.

BRASIL, R.D. et al. Riqueza de plantas e estrutura de quintais familiares no semi - árido do norte mineiro. Revista Brasileira de Biociências, v.5, n. 2, p.864-866, 2007. Disponível em: <http:// www.ufrgs.br/seerbio/ojs/index.php/rbb/article/view/714/599>. Acesso em: 12 nov. 2012.

CARNIELLO, M.A. et al. Quintais urbanos de Mirassol D' OesteMT, Brasil: uma abordagem etnobotânica. Acta Amazonica, v.40, n.3, p.451-470, 2010. Disponível em: <http://www.scielo.br/pdf/ aa/v40n3/05.pdf>. Acesso em: 03 mar. 2013.

FRANCO, F. et al. Etnobotânica: aspectos históricos e aplicativos desta ciência. Caderno de Cultura e Ciência, v.10, n.2, p.17- 
23, 2011. Dsiponível em: <http://periodicos.urca.br/ojs/index.php/ cadernos/article/view/407/pdf >. Acesso em: 12 nov. 2011.

FUCK, S.B. et al. Plantas medicinais utilizadas na medicina popular por moradores da área urbana de Bandeirantes, PR, Brasil. Semina: Ciências Agrárias, v.26, n.3, p.291-296, 2005. Disponível em: <http://www.uel.br/revistas/uel/index.php/ semagrarias/article/view/2304/1984>. Acesso em: 12 nov. 2012.

IBGE. Anuário estatístico. Brasília: Instituto Brasileiro de Geografia e Estatística. Disponível em: <http://www.censo2010. ibge.gov.br/amostra/>. Acesso em: 02 maio 2012.

MARCONI, M.A.; LAKATOS, E.M. Técnicas de Pesquisa: planejamento e execução de pesquisas, amostragens e técnicas de pesquisas, elaboração, análise e interpretação de dados. São Paulo: Atlas, 2002. p.296.

NASCIMENTO, A.P.B. et al. Ecological niche theory: nontraditional urban and rural human populations. Journal of Human Ecology, v.32, n.3, p.175-182, 2010.

NASCIMENTO, A.P.B. et al. Quintais domésticos e sua relação com o estado nutricional de crianças rurais, migrantes e urbanas. Multiciência (ASSER), v.5, p.1-15, 2005. Disponível em: <http:// www.multiciencia.unicamp.br/artigos_05/rede_03_05.pdf >. Acesso em: 03 mar. 2013.

OLIVEIRA, F.C. et al. Avanço nas pesquisas etnobotânicas no Brasil. Acta Botanica Brasilica, v.23, n.2, p.590-605, 2009. Disponível em: <http://www.scielo.br/pdf/abb/v23n2/v23n2a31. pdf>. Acesso em: 13 set. 2012.

PASA, M.C. et al. Estudo Etnobotânico na comunidade de Conceição Açu (alto da bacia do rio Aricá Açu, MT, Brasil). Acta Botanica Brasílica, v.19, n.2, p.195-207, 2005. Disponível em: <http://www.scielo.br/pdf/abb/v19n2/26213.pdf>. Acesso em: 12 nov. 2012.

PAULA, K.B.S.; CRUZ-SILVA, C.T.A. Formas de uso medicinal da babosa e camomila pela população urbana de Cascavel, Estado do Paraná. Acta Science: Health Science, v.32, n.2, p.169-
176, 2010. Disponível em: <http://periodicos.uem.br/ojs/index.php/ ActaSciHealthSci/article/view/6446/6446>. Acesso em: 13 mar. 2013.

PERNA, T.A.; LAMANO-FERREIRA, A.P.N Revisão bibliométrica sobre o cultivo de plantas medicinais em quintais urbanos em diferentes regiões do Brasil (2009-2012). Revista Unopar, v.16, n.1, p.61-67, 2014. Disponível em <http://revistas. unopar.br/index.php/biologicas/article/viewFile/1249/1147> Acesso em 20 mar 2014

QUEIROZ, D.P.N., LAMANO-FERREIRA, A.P.N. Diversidade e Uso de Plantas Cultivadas em Quintais Residenciais Urbanos na Região da Vila Maria, Zona Norte de São Paulo, SP. UNOPAR Científica. Ciências Biológicas e da Saúde, NO PRELO.

SHIVA, V.; DANKELMAN, I. As mulheres e a diversidade biológica: lições do Himalaia indiano. In: GAIFAMI, A. (Org). Cultivando a diversidade: recursos genéticos e segurança alimentar local. Rio de Janeiro: AS-PTA, 1994. p.35-40.

SIVIERO, A. et al. Cultivo de espécies alimentares em quintais urbanos de Rio Branco, Acre, Brasil. Acta Botanica Brasílica, v.25, n3, p.546-553, 2011. Disponível em: <http://www.scielo.br/ pdf/abb/v25n3/06.pdf>. Acesso em: 14 mar. 2013.

SOUZA, M.D. et al. Estudo etnobotânico de plantas medicinais na comunidade São Gonçalo Beira Rio, Cuiabá, MT. Revista Biodiversidade, v.9, n.1, p.91-100, 2010. Disponível em: <http:// periodicoscientificos.ufmt.br/ojs/index.php/biodiversidade/article/ view/104/95>. Acesso em: 14 mar. 2013.

VENDRUSCOLO, G.S.; MENTZ, L.A. Levantamento etnobotânico das plantas utilizadas como medicinais por moradores do bairro Ponta Grossa, Porto Alegre, Rio Grande do Sul, Brasil. Iheringia, Série Botânica, v.61, n. 1-2, p.83-103, 2006.

VIEIRA, M.L.S. et al. Uso popular de plantas medicinais no município de Rio Tinto, PB. In: CONGRESSO DE ECOLOGIA DO BRASIL, São Lourenço, MG. Anais... São Lourenço: Sociedade de Ecologia do Brasil, p. 1-2, 2011. Disponível em <http://www.seb-ecologia.org.br/xceb/resumos/1460.pdf > Acesso em: 06 abr.2013 